\title{
Das Strafrecht in der Krise
}

Eine Untersuchung der Strafbestimmungen in der COVID-19-Verordnung 2 und welche Lehren daraus zu ziehen sind

Gian Ege / David Eschle *

Der Bundesrat hat im Rahmen der Massnahmen zur Bekämpfung des Coronavirus (Covid-19) Strafuorschriften auf Verordnungsebene erlassen und diese als Reaktion auf die sich rasch ändernden Umstände häufig angepasst. Das wirft die Fragen auf, ob der Bundesrat überhaupt Strafvorschriften erlassen darf und welchen Anforderungen diese genügen müssen. Eine Analyse der Strafbestimmungen in der COVID19-Verordnung 2 zeigt, dass sich diese auf eine - wenn auch diskussionswürdige Grundlage stützen können. Die konkrete Ausgestaltung verletzt allerdings teilweise das Verhältnismässigkeitsprinzip und das strafrechtliche Bestimmtheitsgebot. Ausserdem vermag die Notverordnungskompetenz des Bundesrates die Anwendung des Ordnungsbussenverfahrens auf gewisse Übertretungen nicht zu decken. Insgesamt ist es angezeigt, dass der bundesrätliche Umgang mit den Strafvorschriften überdacht wird, um für zukünftige Krisensituationen die richtigen Schlüsse zu ziehen.

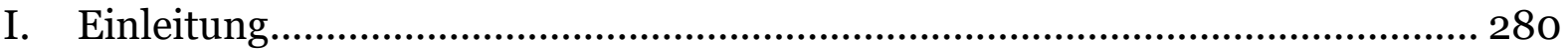

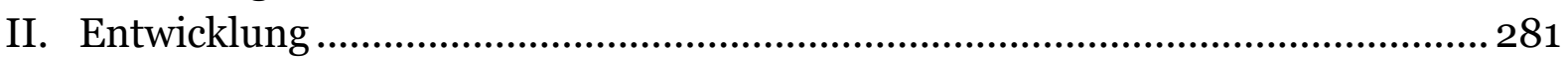

III. Darf der Bundesrat Strafbestimmungen erlassen? ..............................................282

IV. Analyse der Strafbestimmungen ..................................................................28

1. Vergehen (Art. 1of Abs. 1) ..............................................................................28

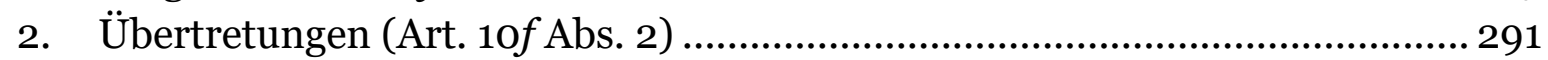

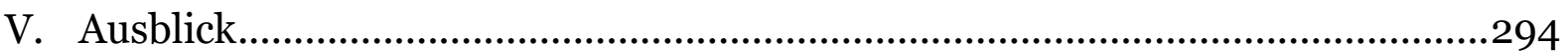

Zitiervorschlag: Gian Ege/David Eschle, Das Strafrecht in der Krise, Eine Untersuchung der Strafbestimmungen in der COVID-19-Verordnung 2 und welche Lehren daraus zu ziehen sind, in: sui-generis 2020, S. 279

URL: $\quad$ sui-generis.ch/137

DOI: $\quad$ https://doi.org/10.21257/sg.137

* Dr. iur. Gian Ege (gian.ege@rwi.uzh.ch), Oberassistent für Strafrecht und Strafprozessrecht, Universität Zürich. David Eschle, MLaw (david.eschle@rwi.uzh.ch), wissenschaftlicher Mitarbeiter am Lehrstuhl für Strafrecht, Strafprozessrecht, Wirtschafts- und Verwaltungsstrafrechts von Prof. Dr. iur. Marc Thommen, Universität Zürich. 


\section{Einleitung}

1 Das Jahr 2020 wird als Jahr der Pandemie in Erinnerung bleiben. $\mathrm{Zu}$ Beginn des Jahres breitete sich ein neuartiges Coronavirus (SARS-CoV-2) und die dadurch verursachte Lungenkrankheit (Covid-19) weltweit rasant aus und wurde von der World Health Organization (WHO) am 11. März 2020 zur Pandemie erklärt. ${ }^{1}$ Auch in der Schweiz verbreitete sich das Virus ab Ende Februar 2020 stark. ${ }^{2}$ Als Reaktion rief der Bundesrat am 28. Februar 2020 die besondere Lage im Sinne des Epidemiengesetzes (EpG) ${ }^{3}$ aus.4 Diese Einschätzung wurde am 16. März 2020 revidiert und die Situation zur ausserordentlichen Lage heraufgestuft.5 Zur Eindämmung der Virusverbreitung sowie zur Abfederung ihrer Auswirkungen hat der Bundesrat zahlreiche Verordnungen erlassen. Die rasch ändernden Umstände unterwarfen diese Regelungswerke einem steten Wandel, was $\mathrm{zu}$ verschiedenen rechtlichen Unklarheiten führte. Mit deren wissenschaftlichen Aufarbeitung beschäftigt sich mittlerweile eine Vielzahl von Publikationen. ${ }^{6}$ Vergleichsweise geringe Be-

$1 \overline{\text { WHO, WHO Director-General's opening remarks }}$ at the media briefing on COVID-19, 11. März 2020.

2 Schweizerische Eidgenossenschaft, COVID-19 in der Schweiz.

3 Bundesgesetz über die Bekämpfung übertragbarer Krankheiten des Menschen vom 28. September 2012 (Epidemiengesetz, EpG; SR 818.101).

4 Medienmitteilung des Bundesrats vom 28. Februar 2020 (Coronavirus: Bundesrat verbietet grosse Veranstaltungen).

5 Medienmitteilung des Bundesrats vom 16. März 2020 (Coronavirus: Bundesrat erklärt die «ausserordentliche Lage» und verschärft die Massnahmen).

6 Z.B. Giovanni Biaggini, «Notrecht» in Zeiten des Coronavirus, ZBl 5/2020, S. 239 ff.; Florian Brunner/Martin Wilhelm/Felix Uhlmann, Das Coronavirus und die Grenzen des Notrechts, AJP 2020, S. 685 ff.; Andreas Kley, "Ausserordentli- achtung erhielt dabei der Umstand, dass der Bundesrat in seinen Verordnungen auch Strafvorschriften erliess.7 Eine Untersuchung dieser Normen drängt sich jedoch auf, da einerseits ungeklärt ist, ob der Bundesrat diese Bestimmungen überhaupt so hätte erlassen dürfen, und die Straftatbestände andererseits selbst grossen Klärungsbedarf aufweisen.

2 Dieser Beitrag zeichnet den Erlass sowie die häufigen und raschen Änderungen der Strafvorschriften nach (II.). Anschliessend wird beleuchtet, ob der Bundesrat überhaupt Strafvorschriften erlassen darf und welchen Anforderungen diese genügen müssen (III.). Die Strafbestimmungen der Verordnungen werden sodann ausführlich auf ihre Rechtmässigkeit hin untersucht (IV.). Aufgrund der daraus gewonnenen Erkenntnisse wird abschliessend aufgezeigt, welche Lehren für eine ähnliche Situation in der Zukunft zu ziehen sind (V.). che Situationen verlangen nach ausserordentlichen Lösungen.», ZBl 121/2020, S. 268 ff.; Andreas Stöckli, Regierung und Parlament in Pandemiezeiten, ZSR 139/2020, Sondernummer «Pandemie und Recht», S. 9 ff.; Emanuel Georg Tschannen, Das Corona-Massnahmenpaket des Bundesrats, Jusletter vom 14. April 2020; Damian Wyss, Sicherheit und Notrecht, Jusletter vom 25. Mai 2020; Andreas Zünd/Christoph Errass, Pandemie - Justiz - Menschenrechte, ZSR 139/2020, Sondernummer «Pandemie und Recht», S. $69 \mathrm{ff}$.

7 Vgl. allerdings Annika Burrichter/Moritz Vischer, Der Vergehenstatbestand nach Art. 1of Abs. 1 der COVID-19-Verordnung 2, forumpoenale online first 2020. 


\section{Entwicklung}

3 Mit der am 28. Februar 2020 in Kraft getretenen Verordnung über Massnahmen zur Bekämpfung des Coronavirus ${ }^{8}$ verbot der Bundesrat Grossveranstaltungen. ${ }^{9}$ In dieser Verordnung waren keine Strafvorschriften vorgesehen. Da sich die Massnahme auf Art. 6 i.V.m. Art. 40 EpG stützte, hätte eine Verletzung des Veranstaltungsverbots eine Bestrafung nach Art. 83 lit. j EpG nach sich ziehen können. ${ }^{10}$ Am 13. März 2020 wurde diese Verordnung durch die Verordnung 2 über Massnahmen zur Bekämpfung des Coronavirus (COVID-19) ${ }^{11}$ abgelöst. Veranstaltungen ab 100 Teilnehmenden wurden verboten. ${ }^{12}$ Gesonderte Strafvorschriften waren nicht vorgesehen, eine Strafbarkeit konnte sich weiterhin aus dem EpG ergeben.

Mit der Deklarierung der ausserordentlichen Lage wurde die Verordnung am 16. März 2020 angepasst. Öffentliche

8 Verordnung über Massnahmen zur Bekämpfung des Coronavirus (COVID-19) vom 28. Februar 2020 (SR 818.101.24).

9 Art. 2 Verordnung über Massnahmen zur Bekämpfung des Coronavirus.

10 Benjamin Märkli, Die «Corona-Verordnung» des Bundesrats vom 28. Februar 2020, Jusletter vom 9. März 2020, Rz. 23; vgl. auch Botschaft vom 3. Dezember 2010 zur Revision des Bundesgesetzes über die Bekämpfung übertragbarer Krankheiten des Menschen (Epidemiengesetz, EpG) (BBl 2011 311), S. 363.

11 Verordnung 2 über Massnahmen zur Bekämpfung des Coronavirus (COVID-19) vom 13. März 2020 (COVID-19-Verordnung 2; SR 818.101.24). Die folgenden Ausführungen beziehen sich auf die Verordnung nach den Änderungen vom 27. Mai 2020 (AS 20201815 ) mit Stand am 6. Juni 2020. Wird auf eine ältere Version abgestellt, ist in Klammern das Datum der letzten Änderungen sowie die Fundstelle in der AS angegeben. Ausserdem wird auf die genaue Bezeichnung der Verordnung verzichtet. Artikel, die ohne nähere Angaben genannt werden, sind immer solche aus der COVID-19-Verordnung 2.

12 Art. 6 (13. März 2020, AS 2020 773). und private Veranstaltungen wurden gänzlich verboten und mittels als Vergehen ausgestalteter, besonderer Strafvorschrift unter Strafe gestellt. ${ }^{13}$ Mit Inkrafttreten am 21. März 2020 wurde ein Verbot von öffentlichen Menschenansammlungen von mehr als 5 Personen in die Verordnung aufgenommen. ${ }^{14}$ Der Verstoss dagegen wurde als Übertretung eingestuft und gleichzeitig wurde für die Ahndung das Ordnungsbussenverfahren vorgesehen. ${ }^{15}$ Als weitere Übertretungen wurden ab dem 26. März 2020 die nicht bewilligte Ausfuhr von Schutzausrüstung16 - per 3. April 2020 erweitert auf wichtige medizinische Güter ${ }^{17}-$, ab dem 2. April 2020 der Verstoss gegen die Einschränkungen des grenzüberschreitenden Personenverkehrs ${ }^{18}$ sowie ab dem 17. April 2020 der Verstoss gegen das Verbot des Einkaufstourismus ${ }^{19}$ in die Verordnung aufgenommen. Letztere zwei wurden ebenfalls dem Ordnungsbussenverfahren unterstellt. ${ }^{20}$

5 Ab dem 27. April 2020 begann der Bundesrat, die Massnahmen schrittweise zu lockern $^{21}$ und gewisse Veranstaltungen wurden wieder zugelassen. ${ }^{22}$ Per 30 . Mai 2020 galt das Versammlungsverbot nur noch für Ansammlungen von mehr als

13 Art. 6 und Art. 10d (16. März 2020, AS 2020 783).

14 Art. 7c (20. März 2020, AS 2020 863).

15 Art. 1od Abs. 3 (20. März 2020, AS 2020 863).

16 Art. $10 f$ Abs. 2 lit.b (25. März 2020, AS 2020 1065).

17 Art. $10 f$ Abs. 2 lit. b (3. April 2020, AS 2020 1155).

18 Art. $10 f$ Abs. 2 lit. c (1. April 2020, AS 2020 1137).

19 Art. $10 f$ Abs. 2 lit. d, Abs. 4 und 5 (16. April 2020, AS 2020 1245).

20 Art. $10 f$ Abs. 4 und 5 (16. April 2020, AS 2020 1245).

21 Vgl. Art. 6 Abs. 2 und 3 (16. April 2020, AS 2020 1249) sowie Art. 6 Abs. 2 und 3 (29. April 2020, AS 20201401 und 8. Mai 2020, AS 2020 1499).

22 Art. 6 Abs. 3 lit. k (20. Mai 2020, AS 2020 1751). 
30 Personen. ${ }^{23}$ Damit einhergehend fielen die entsprechenden Handlungen aus dem Anwendungsbereich der Strafvorschriften. Mit Inkraftsetzung am 6. Juni 2020 wurden die Strafbestimmungen der Lockerung angepasst und inhaltlich präzisiert. ${ }^{24}$ Das Veranstaltungsverbot, das Versammlungsverbot und die entsprechenden Strafbestimmungen gelten voraussichtlich bis zum 5. Juli 2020. ${ }^{25}$

praktisch allen zahlreich neben der COVID-19-Verordnung 2 im Zusammenhang mit der Corona-Pandemie erlassenen Verordnungen ${ }^{26}$ sind keine Strafvorschriften enthalten. Einzige Ausnahme bildet Art. 23 der COVID-19-Solidarbürgschaftsverordnung 27.28

\section{Darf der Bundesrat Strafbestimmungen erlassen?}

7 Zunächst stellt sich die Frage, ob der Bundesrat überhaupt dazu ermächtigt war, in der COVID-19-Verordnung 2 Strafbestimmungen zu erlassen und insbesondere Freiheitsstrafen anzuordnen,

23 Art. 7c Abs. 1 COVID-19-Verordnung 2.

24 Art. 10f COVID-19-Verordnung 2.

25 Art. 12 Abs. 12 COVID-19-Verordnung 2.

26 Siehe die chronologische Darstellung (Stand: 29. April 2020) bei Brunner/Wilhelm/Uhlmann (Fn. 6), S. 687; vgl. auch Tschannen (Fn. 6), Rz. 7 ff.

27 Verordnung zur Gewährung von Krediten und Solidarbürgschaften in Folge des Coronavirus vom 25. März 2020 (SR 951.261).

28 Mit Busse bis zu 100 ooo Franken wird bestraft, «wer vorsätzlich mit falschen Angaben einen Kredit nach dieser Verordnung erwirkt oder die Kreditmittel in Abweichung von Artikel 6 Absatz 3 verwendet». Dieser Tatbestand wird vorliegend nicht näher behandelt, s. dazu Benjamin Märkli/Moritz Gut, Missbrauch von Krediten nach Covid-19-Solidarbürgschaftsverordnung, AJP 6/2020, S. 722 ff. sowie Edy Salmina, Le disposizioni penali previste dall'Ordinanza federale sulle fideuissioni solidali Covid-19, Novità fiscali 3/2020, S. $103 \mathrm{ff}$. oder ob dies der Bundesversammlung als Gesetzgeberin vorbehalten gewesen wäre.

8 Bei Übertretungen ist Art. $1 \mathrm{StGB}^{29} \mathrm{Ge}-$ nüge getan, wenn die Busse in Verordnungen der Exekutive, die sich im Rahmen von Verfassung und Gesetz halten, angedroht ist. $3^{\circ}$ Für Freiheitsstrafen verlangen Bundesgericht und Lehre demgegenüber eine klare Grundlage in einem formellen Gesetz (so explizit Art. 31 Abs. 1 BV $\left.{ }^{31}\right) .{ }^{2}$ Teilweise wird ein Gesetz im formellen Sinn auch bei Geldstrafen gefordert.33 Notverordnungen, die der Bundesrat unmittelbar gestützt auf die Verfassung erlässt, stellen eine Ausnahme dar: Hier können Verstösse unter Umständen auch mit Freiheitsstrafe sanktioniert werden. 34 So beurteilte das Bundesgericht die Androhung von Freiheitsstrafen bei Missachtung des Waffentragverbots für jugoslawische Staatsangehörige, vom Bundesrat direkt auf Art. 102 Ziff. 8-10 aBV (heute Art. 184 Abs. 3 und Art. 185 Abs. 3 BV) gestützt, als verfassungskonform.35 Der Bundesrat könne in Notverordnungen Gefängnis-

$29 \overline{\text { Schweizerisches Strafgesetzbuch vom 21. Dezem- }}$ ber 1937 (StGB; SR 311.0).

3о BGE 96 I 24 E. 3a; vgl. EJPD, Gesetzgebungsleitfaden, 4. Aufl., Bern 2019, Rz. 891.

31 Bundesverfassung der Schweizerischen Eidgenossenschaft vom 18. April 1999 (BV; SR 101).

32 BGE 124 IV 23 E. 1; BGE 112 Ia 107 E. 3b; Wolfgang Wohlers, in: Wohlers/Godenzi/Schlegel, Schweizerisches Strafgesetzbuch, Handkommentar, 4. Aufl., Bern 2020, Art. 1 N 3 (zit. HK StGBBearbeiterIn).

33 Peter Popp/Anne Berkemeier, in: Niggli/Wiprächtiger (Hrsg.), Strafrecht, Basler Kommentar, 4. Aufl., Basel 2019, Art. 1 N 28 (zit. BSK StGB-BearbeiterIn).

34 Gilbert Kolly, Selbständige Verordnungen als Grundlage für Freiheitsstrafen, SJZ 89/1993, S. 352 ff., 354; Stefan Trechsel/Marc JeanRichard-dit-Bressel, in: Trechsel/Pieth (Hrsg.), Schweizerisches Strafgesetzbuch, Praxiskommentar, 3. Aufl., Zürich/St. Gallen 2018, Art. 1 N 13.

35 BGE 123 IV 29. 
strafen (heute Freiheitsstrafen bis zu drei Jahren) androhen, wenn diese dem Unwert, der in der Missachtung der bundesrätlichen Anordnungen und Verbote liege, angemessen sind. ${ }^{36}$ Im Ergebnis stellt die Rechtsprechung zum Waffentragverbot für jugoslawische Staatsangehörige an Strafnormen die gleichen Anforderungen wie an andere Bestimmungen des konstitutionellen «Notrechts»:37 Liegt eine Situation gemäss Art. 185 Abs. 3 BV vor, ${ }^{8}$ können Strafbestimmungen erlassen werden, wenn dies dringend notwendig ist, die Strafe zur Durchsetzung der Verordnung unbedingt erforderlich und im Vergleich zum Tatunrecht verhältnismässig ist.39 Bei der Androhung von freiheitsentziehenden Sanktionen in Notverordnungen muss indessen grosse Zurückhaltung geübt werden. ${ }^{40}$

9 Wie steht es also um die Bestimmungen in Art. 10f? Nicht einheitlich beantwortet wird bislang die für die Ermächtigung wichtige Frage, ob sich die bundesrätlichen Massnahmen und Anordnungen in der Verordnung (und damit auch die Strafnormen) materiell auf die Verfassung und/oder auf das EpG stützen. ${ }^{41}$

36 BGE 123 IV 29 E. 4c.

37 Thomas Gächter, Selbstständiges Verordnungsrecht des Bundesrates und Gesetzesvorbehalt für Freiheitsstrafen, in: Ackermann (Hrsg.), Strafrecht als Herausforderung, Zürich 1999, S. 231 ff., 240 f.; Stöckli (Fn. 6), S. 25.

38 Eine schwere Störung der öffentlichen Ordnung oder der inneren bzw. äusseren Sicherheit muss eingetreten sein oder unmittelbar drohen. Ausserdem sind die Verordnungen zu befristen.

39 Gächter (Fn. 37), S. 241; David Rechsteiner, Recht in besonderen und ausserordentlichen Lagen, Diss. St. Gallen, Zürich/St. Gallen 2016, S. 175 .

40 Gächter (Fn. 37), S. 241; vgl. auch Guido Jenny, Die strafrechtliche Rechtsprechung des Bundesgerichts im Jahre 1997, ZBJV 134/1998, S. 606 ff., 609.

41 Stöckli (Fn. 6), S. 21 f., geht davon aus, dass sich die Primärmassnahmen auf Art. 7 EpG stützen,
Der Bundesrat geht von Letzterem aus: Im Ingress der Verordnung figurieren Art. 184 Abs. 3 und Art. 185 Abs. 3 BV nach einem dreitägigen Intermezzo im März 2020 nicht mehr als Ermächtigungsgrundlagen. ${ }^{2}$ Die COVID-19Verordnung 2 verweist seit der Ausrufung der ausserordentlichen Lage am 16. März - als einzige der CoronaNotverordnungen - auf Art. 7 EpG.43 Dieser ermächtigt den Bundesrat in einer ausserordentlichen Lage, für das ganze Land oder einzelne Landesteile die «notwendigen Massnahmen» anzuordnen.44 Den Ermächtigungsgrundlagen, die im Ingress von bundesrechtlichen Erlassen angegeben werden, kommt jedoch keine normative Kraft zu.45 Der Bundesrat kann mit anderen Worten auch konstitutionelles Notrecht erlassen, ohne dies explizit zu sagen. Aus den beiden möglichen Grundlagen - Art. 185 Abs. 3

wohl auch Biaggini (Fn. 6), S. 262 f.; a.M. Brunner/Wilhelm/Uhlmann (Fn. 6), S. 696 f.; Burrichter/Vischer (Fn. 7); differenzierend in Bezug auf die Strafbestimmungen Zünd/Errass (Fn. 6), S. 89.

42 Vom 13. bis 16. März 2020, vgl. Ingress der COVID-19-Verordnung 2 (von 13. März 2020, AS 2020 773, bis 16. März 2020, AS 2020 783).

43 Mit der Änderung vom 18. März 2020 (AS 2020 841) wurden zwei weitere Rechtsgrundlagen in den Ingress der Verordnung aufgenommen: Anhang I Art. 5 des Abkommens vom zwischen der Schweizerischen Eidgenossenschaft einerseits und der Europäischen Gemeinschaft und ihren Mitgliedstaaten andererseits über die Freizügigkeit 21. Juni 1999 (FZA; SR 0.142.112.681) sowie Art. 28 der EU-Verordnung 2016/399 des Europäischen Parlaments und des Rates vom 9. März 2016 über einen Gemeinschaftskodex für das Überschreiten der Grenzen durch Personen (Schengener Grenzkodex); beide wirken sich nicht auf die innerstaatliche Legitimation des Bundesrats aus.

44 Damit ist auch der Erlass von Verordnungen gemeint, anders könnte der Bundesrat keine generell-abstrakten Anordnungen für das ganze Land treffen; vgl. Stöckli (Fn. 6), S. 20.

45 BGE 144 II 454 E. 4.3.1; Rechsteiner (Fn. 39), Rz. 511; Zünd/Errass (Fn. 6), S. 88. 
BV und Art. 7 EpG - resultieren für die Strafnormen der Verordnung zwei Szenarien.

Zunächst ist denkbar, dass sich die Strafbestimmungen direkt auf die Notverordnungskompetenz nach Art. 185 Abs. 3 BV stützen. Für diese Auslegung spricht in erster Linie die Botschaft zum noch jungen EpG, das erst Anfang 2016 in Kraft trat. Ihr zufolge ist Art. 7 EpG nur ein deklaratorischer Verweis auf Art. 185 Abs. 3 BV und verankert das konstitutionelle Notrecht auf Gesetzesstufe. 46 Art. 7 EpG schafft somit keine neuen Kompetenzen, sondern wiederholt lediglich diejenigen der Verfassungsbestimmung.47 Auch eine verfassungskonforme Auslegung führt zu diesem Ergebnis. Die Entscheidung, welche Rechtsetzungsbefugnisse dem Bundesrat in Zeiten der Not zukommen, wurde im Grundsatz durch den Verfassungsgeber gefällt. 48 Dieser entschied sich gegen eine umfassende Staatsnotstandsklausel 49 und statuierte eine Pflicht zur Befristung von Notverordnungen (Art. 185 Abs. 3 BV). Der Gesetzgeber unterstellte solche überdies einem Überführungsmechanismus: Der Bundesrat muss dem Parlament innert sechs Monaten einen Entwurf für ein Bundesgesetz oder eine Notverordnung der Bundesversammlung unterbreiten, ansonsten tritt die bundesrätliche Verordnung ausser Kraft (vgl. Art. $7 d$ Abs. 2 RVOG50).51 Art. 7 EpG auferlegt dagegen

46 Botschaft EpG (Fn. 10), S. 334, 337 sowie 364 f.

47 Brunner/Wilhelm/Uhlmann (Fn. 6), S. 693.

48 So auch Zünd/Errass (Fn. 6), S. 89 f., allerdings mit gegenteiligem Schluss.

49 Biaggini (Fn. 6), S. 242.

50 Regierungs- und Verwaltungsorganisationsgesetz vom 21. März 1997 (RVOG; SR 172.010).

$51 \mathrm{Zu}$ den daraus resultierenden Fragen in Bezug auf die Corona-Notverordnungen Biaggini (Fn.6), S. 251 ff. sowie Stöckli (Fn. 6), S. 48. keine Pflicht, Massnahmen zu befristen oder in ordentliches Recht zu überführen. ${ }^{2}$ Auch der Rechtsschutz wäre bei einer Abstützung auf Art. 7 EpG wegen Art. 190 BV im Vergleich zum verfassungsunmittelbaren Notrecht eingeschränkt.53 Vor diesem Hintergrund scheint es wenig plausibel und mit dem Notrechtsinstitut der BV schwer vereinbar, dass die Bundesversammlung der Regierung mit dem offen formulierten Art. 7 EpG eigenständige - also neben den vom Verfassungsgeber vorgesehenen - (Not-)Rechtsetzungsbefugnisse verleihen wollte. 54

11 Handelt es sich bei Art. $10 f$ um eine «echte» Notverordnungsbestimmung i.S.v. Art. 185 Abs. 3 BV, hätte diese den allgemeinen Verfassungsprinzipien $\mathrm{zu}$ genügen, also insbesondere dem Rechtsgleichheitsgebot und dem Verhältnismässigkeitsprinzip. 55 Es wäre zu prüfen, ob die Strafe zur Durchsetzung der vom Bundesrat aufgestellten Verhaltensnormen und Verbote erforderlich ist und die angedrohten Strafen verhältnismässig sind zum Unrechtsgehalt des Normbru-

52 So auch Biaggini (Fn. 6), S. 265; Brunner/Wilhelm/Uhlmann (Fn. 6), S. 694; a.M. Stöckli (Fn. 6), S. 48, der postuliert, Art. $7 d$ RVOG auch auf Notverordnungen anzuwenden, die gestützt auf Art. 7 EpG erlassen wurden; ebenso Wyss (Fn. 6), Rz. 15; in diese Richtung deutet auch die Botschaft EpG (Fn. 10), S. 365 f., wobei Art. $7 d$ RVOG zu jenem Zeitpunkt noch nicht in Kraft war.

53 Brunner/Wilhelm/Uhlmann (Fn. 6), S. 697; eine abstrakte Normenkontrolle ist auf jeden Fall unzulässig, vgl. Urteil des Bundesgerichts 2C_280/2020 vom 15. April 2020 E. 2.2.

54 Ebenso Brunner/Wilhelm/Uhlmann (Fn. 6), S. 694; a.M. Biaggini (Fn. 6), S. 263 f.; Kley (Fn. 6), S. 273.

55 Giovanni Biaggini, Bundesverfassung der Schweizerischen Eidgenossenschaft, 2. Aufl., Zürich 2017, Art. 185 N 10a; Ralph Trümpler, Notrecht, Diss. Zürich, Zürich/Basel/Genf 2012, Rz. 310 ff. 
ches. ${ }^{6}$ Was der Gesetzgeber in Art. 82 und 83 EpG bereits unter Strafe gestellt hat, wäre Richtschnur für die Verhältnismässigkeitsprüfung. 57 Die Anordnungen in der Verordnung dürfen nach bundesgerichtlicher Rechtsprechung nämlich grundsätzlich nicht im Widerspruch zu Erlassen der Bundesversammlung stehen. 58

Das zweite mögliche Szenario ist, dass die Strafbestimmungen der COVID-19Verordnung 2 ihre Grundlage direkt in Art. 7 EpG finden. Die Lehre sieht darin teilweise eine eigenständige epidemiologische Ermächtigungsnorm für bundesrätliches Notrecht.59 Wie weit diese Kompetenz reicht und welche "Massnahmen» sie genau umfassen soll, ist im Einzelnen ungeklärt. ${ }^{60}$ Teilweise wird postuliert, Art. 7 EpG stelle eine im Vergleich $\mathrm{zu}$ Art. 185 Abs. 3 BV weitergehende Ermächtigung dar, ${ }^{61}$ beispielsweise indem er auch finanzielle Leistungen des Bundes zulässt, die den Rahmen des verfassungsunmittelbaren Notrechts sprengen. ${ }^{62}$

56 Gächter (Fn. 37), S. 243.

57 S. dazu unten IV.1.a.

58 BGE 123 IV 29 E. 3a S. 34. In der Corona-Krise hat sich zwar gezeigt, dass diese Regel nicht absolut gelten kann und der Bundesrat unter Umständen auch von Bundesgesetzen abweichendes Recht setzen können muss, s. für Beispiele und zum Ganzen Brunner/Wilhelm/Uhlmann (Fn. 6), S. $696 \mathrm{ff}$. Von den gesetzgeberischen Wertungen im EpG, das gerade die Bekämpfung von Epidemien zum Ziel hat, dürfte allerdings nur sehr zurückhaltend divergiert werden.

59 So insb. Biaggini (Fn. 6), S. 264; Kley (Fn. 6), S. 272; in Bezug auf Primärmassnahmen auch Stöckli (Fn. 6), S. 47; s. auch IV.1.a.

6o S. z.B. Zünd/Errass (Fn. 6), S. 83 ff.; Biaggini (Fn. 6), S. $260 \mathrm{ff}$.

${ }^{61}$ Biaggini (Fn. 6), S. 263; Kley (Fn. 6), S. 272 ff.; a.M. Stöckli (Fn. 6), S. 20 f.

62 Biaggini (Fn. 6), S. $261 \mathrm{f}$.
13 In Bezug auf den Erlass der Strafnormen in Art. $10 f$ gehen verschiedene Autoren davon aus, dass Art. 7 EpG nur eine wacklige oder keine genügende Ermächtigungsbasis bildet. 63 Sie argumentieren insbesondere, die Strafbestimmungen verletzten das Verhältnismässigkeitsprinzip64und die Straftatbestände des EpG hätten abschliessenden Charakter. ${ }^{65}$ Letzteres überzeugt nicht: In einer normalen Lage können kantonale Behörden insbesondere Massnahmen gegenüber der Bevölkerung erlassen (Art. 40 EpG). In einer besonderen Lage ist der Bundesrat dazu ermächtigt (Art. 6 Abs. 2 lit. b i.V.m. Art. 40 EpG). Einen Verstoss gegen solche Massnahmen stellte der Gesetzgeber als Übertretung (Art. 83 Abs. 1 lit. j EpG) unter Strafe. ${ }^{66}$ Anders ist die Situation demgegenüber in einer ausserordentlichen Lage: Der Bundesrat kann alle Massnahmen anordnen, die notwendig sind und den Zwecken des EpG67 und der Bekämpfung der Epidemie dienen. ${ }^{68}$ Die Strafbestimmungen des EpG können hier wegen des strengen Analogieverbots $^{69}$ keine Anwendung finden. Gleichzeitig ist es erforderlich, dass der Bundesrat die Massnahmen in einer ausserordentlichen Lage wie diejenigen des Gesetzes unter Strafe stellen kann.

63 Niggli Marcel Alexander, Bundesrat darf keine Strafen erlassen, NZZ vom 16. April 2020, S. 7; Stöckli (Fn. 6), S. 25; Zünd/Errass (Fn. 6), S. 89.

64 Dazu eingehend IV.1.b.

65 Niggli (Fn. 63), S. 7; Stöckli (Fn. 6), S. 25; David Zollinger, Nulla poena sine lege oder die rechtliche Seite der Virusbekämpfung, Inside Paradeplatz vom 30. März 2020.

66 Rechsteiner (Fn. 39), Rz. 890; Zünd/Errass (Fn. 6), S. 84; Botschaft EpG (Fn. 10), S. 363, Tabelle 3 sowie 365 .

67 Vgl. Art. 2 EpG.

68 Kley (Fn. 6), S. 273; Trümpler (Fn. 55), Rz. 181; vgl. auch Botschaft EpG (Fn. 10), S. 363, Tabelle 3.

69 BSK StGB-Popp/Berkemeier, Art. 1 N 31; HK StGB-Wohlers, Art. 1 N 13. 
14 Geht man davon aus, dass es sich bei den Strafbestimmungen in der Verordnung um Massnahmen in einer ausserordentlichen Lage nach Art. 7 EpG handelt, hat man es nicht mehr mit eigentlichen Notverordnungsbestimmungen zu tun. Damit gewinnt die Frage an Bedeutung, inwieweit die Anforderung an ein Gesetz im formellen Sinn gemäss Art. 1 StGB erfüllt sind: Strafvorschriften in bundesrätlichen Notverordnungen, die sich direkt auf die Verfassung stützen, können den Anforderungen von Art. 1 StGB genügen. Ob dies auch für Strafnormen in Verordnungen gelten kann, die sich auf eine (wenn auch sehr umfassende) Delegation in einem Gesetz stützen, ist dagegen zweifelhaft. Eine weitere Konsequenz dürfte sein, dass sich der Bundesrat stärker an der im EpG vorgenommenen Abwägung orientieren muss, $o b$ und wie Verstösse gegen epidemiologische Massnahmen zu bestrafen sind, als dies bei einer Abstützung auf Art. 185 Abs. 3 BV der Fall wäre. ${ }^{\circ}$ Freilich ist die Regierung nicht davon entbunden, sich an die grundlegenden Bestimmungen der Verfassung zu halten, insbesondere an das Verhältnismässigkeitsprinzip. ${ }^{71}$

Die Frage, was materiell Grundlage für die Strafbestimmungen in der COVID19-Verordnung 2 bildet, ist zumindest in der ausserordentlichen Lage von untergeordneter Bedeutung: Dann dürften nämlich die Voraussetzungen für die Anwendung von Art. 185 Abs. 3 BV erfüllt sein (davon geht der Bundesrat bei

70 S. IV.1.b; in diesem Sinne auch Zünd/Errass (Fn. 6), S. 89.

${ }_{71}$ Zünd/Errass (Fn. 6), S. 84; unklar in dieser Hinsicht BGE 131 II 670 E. 3.3, wonach die inhaltlichen Schranken für Massnahmen nach Art. 10 aEpG (dem Vorgängerartikel von Art. 7 EpG) nicht abstrakt bestimmt werden können. zahlreichen anderen Corona-Notverordnungen aus)72: Die Verordnung ist gemäss deren Art. 12 Abs. 3 befristet und die sechsmonatige Frist zur Überführung in ordentliches Recht nach Art. $7 d$ Abs. 2 RVOG läuft erst im Frühherbst ab. Soweit Art. 7 EpG als Grundlage für den Erlass von Strafbestimmungen nicht ausreicht, kann sich der Bundesrat deshalb auf Art. 185 Abs. 3 BV stützen.73 Die Strafnormen wären rechtmässig, wenn sie sich an die allgemeinen Verfassungsprinzipen halten und der Wahrung der Schutzgüter von Art. 185 Abs. 3 BV (öffentliche Ordnung, innere und äussere Sicherheit) 74 dienen.75

72 Vgl. die Auflistung der 14 Verordnungen bei Biaggini (Fn. 6), Fn. 65; in der Zwischenzeit kamen folgende vier dazu (Stand: 6. Juni 2020): die Verordnung über die Durchführung der kantonalen gymnasialen Maturitätsprüfungen 2020 angesichts der Pandemie des Coronavirus vom 29. April 2020 (COVID-19-Verordnung gymnasiale Maturitätsprüfungen; SR 413.16), die Verordnung über Übergangsmassnahmen zugunsten der Printmedien im Zusammenhang mit dem Coronavirus vom 20. Mai 2020 (Covid-19Verordnung Printmedien; SR 783.03), die Verordnung über Übergangsmassnahmen zugunsten der elektronischen Medien im Zusammenhang mit dem Coronavirus vom 20. Mai 2020 (Covid19-Verordnung elektronische Medien; SR 784.402) sowie die Verordnung über die Abfederung der wirtschaftlichen Auswirkungen der Massnahmen zur Bekämpfung des Coronavirus (Covid-19) auf die institutionelle familienergänzende Kinderbetreuung vom 20. Mai 2020 (Covid-19-Verordnung familienergänzende Kinderbetreuung; SR 862.1).

73 So auch Stöckli (Fn. 6), Fn. 98; a.M. wohl Biaggini (Fn. 6), S. 263, Fn. 113, der vom Vorrang von Art. 7 EpG ausgeht, ohne sich zu den Strafbestimmungen zu äussern; Kley (Fn. 6), S. $271 \mathrm{f}$.

74 Biaggini (Fn. 55), Art. 185 N 10.

75 Dazu IV.1.a. 


\section{Analyse der Strafbestimmungen}

\section{Vergehen (Art. 10f Abs. 1)}

16 Der als Vergehen ausgestaltete Art. $10 f$ Abs. 1 wirft zwei Fragen auf: Ist die Norm verhältnismässig (a) und genügt sie dem Bestimmtheitsgebot (b)?

\section{a) Verhältnismässigkeit}

Art. $10 f$ Abs. 1 stellt in all seinen bisherigen Fassungen Widerhandlungen gegen die Verbote und Einschränkungen von Veranstaltungen und Betrieben in Art. $6 \mathrm{ff}$. unter Strafe. Er hat damit grundsätzlich zum Ziel, Massnahmen zur Eindämmung der Übertragung des Coronavirus strafrechtlich abzusichern und das Unrecht von Verstössen gegen solche Massnahmen abzugelten. Dies dürfte mittelbar nicht nur dem Zweck des EpG dienen, die Verbreitung übertragbarer Krankheiten zu bekämpfen (vgl. Art. 2 Abs. 1 EpG). Auch der Schutz der Gesundheit der Gesamtbevölkerung - ein hochrangiges Gut i.S.v. Art. 185 Abs. 3 BV - dürfte damit angestrebt werden. Als materielle Schranke für den Erlass von Strafnormen in der CoronaKrise bleibt somit das Verhältnismässigkeitsprinzip. ${ }^{76}$ Als Not-Gesetzgeber muss der Bundesrat die Strafnormen so ausgestalten, dass diese abstrakt verhältnismässig sind. Damit erhält er gewisse Gestaltungsspielräume:77 Im Gegensatz zu Interessenabwägungen im Einzelfall, dem wohl wichtigsten Anwendungsfall des Verhältnismässigkeitsprinzips, 78 ist dieses als Maxime in der Rechtsetzung weniger griffig. 79

76 Im Ergebnis ebenso Burrichter/Vischer (Fn. 7).

77 BGE 123 IV 29 E. 3b; BGE 131 II 670 E. 3.1.

78 Biaggini (Fn. 55), Art. 5 N 20.

79 Georg Müller/Felix Uhlmann, Elemente einer Rechtsetzungslehre, 3. Aufl., Zürich 2013, Rn. 52
18 Die Strafbestimmungen des EpG dürften - gerade, wenn sich die Verordnung auf Art. 185 Abs. 3 BV stützen sollte - keine harten Grenzen für diese Prüfung darstellen. Dennoch sind die dem EpG zugrundeliegenden Wertungen des Gesetzgebers der naheliegende Massstab, an dem sich der Bundesrat orientieren muss, wenn er Strafbestimmungen verhältnismässig ausgestalten will. Dies gilt umso mehr, weil der Bundesrat in Notlagen die Aufgaben des Parlaments unter Zeitdruck übernimmt. Er sollte nicht ohne gute Gründe von der Wertung abweichen, die der demokratisch legitimierte Gesetzgeber bereits sorgfältig vorgenommen hat.

19 Das Gesetz stellt die meisten Verstösse gegen epidemiologische Massnahmen gemäss Art. 83 Abs. 1 lit. a-m EpG als Übertretungen unter Strafe. Vergehen sind gemäss Art. 82 Abs. 1 EpG nur die Verletzung der Vorschriften zum Umgang mit Krankheitserregern (Art. 26-28 EpG) sowie Verstösse gegen das Berufsund Tätigkeitsverbot (Art. 38 EpG). Die Unterscheidung knüpft an die Schwere der Widerhandlung an: «Schwere Verletzungen hochrangiger Rechtsgüter werden als Vergehen, weniger schwere Angriffe als Übertretungen sanktioniert.»80 Diese Wertung reflektiert die COVID-19Verordnung 2 höchstens punktuell. Der Charakter der Massnahmen in Art. $6 \mathrm{ff}$. entspricht demjenigen verschiedener Massnahmen des Gesetzes: Art. 40 Abs. 2 EpG sieht wie Art. 6 der Verordnung Veranstaltungsverbote (lit. a) sowie Massnahmen zum Betrieb von privaten Unternehmen und öffentlichen Instituti-

m.H. zur Rechtsprechung; Biaggini (Fn. 55), Art. $5 \mathrm{~N} 20$.

80 Botschaft EpG (Fn. 10), S. 422. 
onen bis hin zur Schliessung (lit. b) vor. Die Pflicht zur Erstellung und zur Einhaltung von Schutzkonzepten, die im Rahmen der Lockerungen einen wesentlichen Teil des Straftatbestands in Art. $10 \mathrm{f}$ ausmacht, erinnert stark an die Verhütungsmassnahmen nach Art. 19 Abs. 2 lit. b EpG. Der Bundesrat kann Betriebe und Veranstalter verpflichten, Präventionsmaterial bereitzustellen und bestimmte Verhaltensregeln einzuhalten, wobei er die Schwere der Erkrankung und die Grösse des Risikos bei der Konkretisierung dieser Massnahmen zu berücksichtigen hat. ${ }^{81}$ Der Gesetzgeber hat Verstösse gegen beide diese Massnahmen nur als Übertretungen unter Strafe gestellt (Art. 83 Abs. 1 lit. c bzw. lit. j EpG). Die Tatbestände in der Verordnung befinden sich mit einer Strafdrohung von bis zu drei Jahren Freiheitsstrafe an der Obergrenze von Vergehen (vgl. Art. 10 Abs. 3 StGB) und werden damit deutlich härter sanktioniert.

Aus den Erläuterungen zur Verordnung geht nicht hervor, wieso der Bundesrat die Strafdrohungen im Vergleich zum EpG teilweise erheblich verschärfte, aber beispielsweise die Fahrlässigkeitsstrafbarkeit ${ }^{82}$ nicht übernahm. Denkbar wäre, dass die Strafandrohungen des EpG dem Bundesrat in der ausserordentlichen Lage zu lasch erschienen. Die vom Gesetzgeber eher im tieferen Bereich angesetzten Bestrafungen sind allerdings in ihrem grundrechtlichen Kontext zu sehen: Die Übertretungstatbestände des EpG knüpfen an Massnahmen an, die teilweise ohnehin schon stark in die Freiheitsrechte

81 Botschaft EpG (Fn. 10), S. 377.

82 Art. 82 Abs. 2 EpG. des Einzelnen eingreifen ${ }^{83}$ - deren Missachtung unter hohe Strafen zu stellen, würde dazu führen, dass der Grundrechtseingriff im Ergebnis unverhältnismässig wäre. Diese Überlegungen dürften auch für die Verbote und Einschränkungen von Versammlungen und wirtschaftlichen Tätigkeiten in Art. 6 ff. der Verordnung gelten, die der Bundesrat in der ausserordentlichen Lage angeordnet hat. In diesem Punkt unterscheidet sich die Situation auch von jener im Entscheid zum Waffentragverbot für jugoslawische Staatsangehörige, in dem sich das Bundesgericht zum bisher einzigen Mal zur Androhung von Freiheitsstrafen in Notverordnungen geäussert und diese als verfassungskonform beurteilt hat. 84 Das Verbot, in der Öffentlichkeit Waffen zu tragen, stellte einen «höchstens geringfügigen Eingriff in die Freiheitsrechte» 85 dar.

21 Weiter ist in der Abwägung zu berücksichtigen, dass Art. $10 f$ Abs. 1 lediglich abstrakt gefährdendes Verhalten unter Strafe stellt ${ }^{86}$ und keine tatsächliche Rechtsgutsverletzung verlangt. Den unter Strafe gestellten Handlungen wird eine typische Gefährlichkeit für die Gesundheit unterstellt. $87 \mathrm{Ob}$ eine Ansteckungsgefahr tatsächlich besteht oder eine Person das Coronavirus in sich trägt, ist für die Strafbarkeit nicht entscheidend, was

$8 3 \longdiv { \text { Bspw. die Quarantäne (Art. } 3 5 \text { i.V.m. Art. } 8 3 }$ Abs. 1 lit. h EpG), die Pflicht, sich ärztlich untersuchen und Proben entnehmen zu lassen (Art. 36 i.V.m. Art. 83 Abs. 1 lit. i EpG) oder die verschiedenen Massnahmen gegenüber der Bevölkerung (Art. 40 i.V.m. Art. 83 Abs. 1 lit. j EpG).

84 BGE 123 IV 29.

85 BGE 123 IV 29 E. 3 b S. 36.

86 A.M. Burrichter/Vischer (Fn. 7), die von einem konkreten Gefährdungsdelikt ausgehen.

87 Vgl. Günter Stratenwerth, Schweizerisches Strafrecht, Allgemeiner Teil 1: Die Straftat, 4. Aufl., Bern 2011, § 9 N 15. 
gerade bei Handlungen, die im Grenzbereich der Strafbarkeit liegen, problematisch ist. Auch vor diesem Hintergrund erscheint die Qualifikation der Verstösse in Art. $10 f$ Abs. 1 als Vergehen unverhältnismässig. ${ }^{88}$

\section{b) Bestimmtheit}

Art. 1 StGB verlangt, dass eine Tat ausdrücklich unter Strafe gestellt sein muss. Das strafrechtliche Bestimmtheitsgebot («nulla poena sine lege certa») verlangt, dass Strafnormen präzise formuliert sein müssen. ${ }^{89}$ Diese Voraussetzung gilt auch im Nebenstrafrecht.90 Das Bestimmtheitsgebot ist in Art. 7 EMRK ausserdem völkerrechtlich verankert.91 Der Grundsatz verlangt, dass Strafvorschriften so formuliert sind, «dass der Bürger sein Verhalten danach richten und die Folgen eines bestimmten Verhaltens mit einem den Umständen entsprechenden Grad an Gewissheit erkennen kann».92 Dies bedeutet allerdings nicht, dass der Gesetzgeber gänzlich auf allgemeine und vage Begriffe verzichten müsste. Sowohl das Bundesgericht als auch der EGMR verlangen keine absolute Bestimmtheit.93 Der verlangte Bestimmtheitsgrad hängt insbesondere ab «von der Vielfalt der zu ordnenden Sachverhalte, von der Komplexität und der Vorhersehbarkeit der im Einzelfall erforderlichen Entscheidung,

88 Kritisch auch Stöckli (Fn. 6); Zünd/Errass (Fn. 6), S. 89; a.M. Burrichter/Vischer (Fn. 7).

89 BGE 141 IV 279 E. 1.3.3; HK StGB-Wohlers, Art. 1 $\mathrm{N} 7$.

90 BGE 141 IV 279 E. 1.3.3.

91 Urteil des EGMR [GK] 9174/02 vom 19. September 2008 (Korbely gegen Ungarn), Ziff. 73; vgl. auch BSK StGB-Popp/Berkemeier, Art. $1 \mathrm{~N} 50$.

92 BGE 141 IV 279 E. 1.3.3; BGE 138 IV 13 E. 4.1; BGE 117 Ia 472 E. 3 e.

93 BGE 138 IV 13 E. 4.1; BGE 132 I 49 E. 6.2; Urteil des EGMR 23372/94 vom 24. Februar 1998 (Larissis u.a. gegen Griechenland). von den Normadressaten, von der Schwere des Eingriffs in Verfassungsrechte und von der erst bei der Konkretisierung im Einzelfall möglichen und sachgerechten Entscheidung»94. Insgesamt wird eine Verletzung des Bestimmtheitsgebots nur in Extremfällen angenommen; nämlich dann, wenn die Strafvorschriften evident unbestimmt sind. 95

23 Besonders problematisch sind Blankettstrafnormen, also Straftatbestände, deren Inhalt erst durch weitere Bestimmungen konkretisiert werden.96 Das Bundesgericht ist der Auffassung, dass grundsätzlich auch sie dem Bestimmtheitsgebot genügen können.97 Wenn die Ermittlung des Normgehalts aber zu aufwendig und kompliziert ist, wird dem Erfordernis der hinreichenden Präzisierung einer Strafnorm nicht mehr entsprochen. ${ }^{8}$

24 Art. $10 f$ Abs. 1 ist eine Blankettstrafnorm. Die Strafbarkeit ergibt sich erst in Kombination mit Art. 6 ff. Zunächst ist problematisch, dass sich der Regelungsgehalt von Art. 6 immer wieder veränderte und teilweise nur für einige Tage in einer Fassung verblieb, was die Nachvollziehbarkeit und Voraussehbarkeit der Strafbarkeit erheblich erschwerte. 99 Daneben bestanden auch inhaltlich Unklarheiten. So war weitgehend unbestimmt, was alles

$94 \overline{\text { BGE } 138 \text { IV } 13}$ E. 4.1; vgl. auch BSK StGBPopp/Berkemeier, Art. $1 \mathrm{~N} 46$.

95 HK StGB-Wohlers, Art. 1 N 10.

96 HK StGB-Wohlers, Art. 1 N 9; vgl. auch BSK StGB-Popp/Berkemeier, Art. $1 \mathrm{~N} 45$.

97 Urteile des Bundesgerichts 6B_866/2016 vom 9. März 2017 E. 5.2; 6B_967/2015 vom 22. April 2016 E. 2.3; 6B_385/2008 vom 21. Juli 2008 E. 3.3.2.

98 HK StGB-Wohlers, Art. $1 \mathrm{~N} 9$.

99 Vgl. auch Burrichter/Vischer (Fn. 7). 
als verbotene Veranstaltung nach Art. 6 galt. Obschon in den Erläuterungen zur Verordnung etwa Konzerte, Kongresse, Sportveranstaltungen, Demonstrationen, Quartierfeste und Generalversammlungen als Beispiele für verbotene Veranstaltungen genannt wurden, ${ }^{100}$ waren die Grenzen des Verbots unscharf. So sollten «Veranstaltungen im kleinen privaten Rahmen»101 nicht erfasst sein. ${ }^{102}$ Wie weit der kleine private Rahmen reichte, blieb unklar. Die Unbestimmtheit des Veranstaltungsbegriffs zeigt sich auch am Beispiel von Demonstrationen: In den Erläuterungen wurden sie explizit als verbotene Veranstaltungen aufgeführt. ${ }^{103}$ Nachdem das absolute Demonstrationsverbot vermehrt in die Kritik geraten war, sah sich der Bundesrat zu einer Änderung veranlasst. Es wurde jedoch nicht die Verordnung angepasst, sondern an einer Pressekonferenz erklärt, dass die Juristen des Bundes zu einer Neubeurteilung gekommen seien und Demonstrationen nicht mehr als Veranstaltungen zählten. Unter Einhaltung des Sicherheitsabstands sei das Demonstrieren demnach möglich. ${ }^{104}$ Dieses fast saloppe Vorgehen der Behörden verdeutlicht die Unschärfe des Veranstaltungsbegriffs.

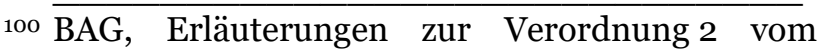
13. März 2020 über Massnahmen zur Bekämpfung des Coronavirus (COVID-19-Verordnung 2) (Version vom 28. Mai 2020; aktualisiert am 29. Mai 2020), S. 21.

101 BAG, Erläuterungen zur Verordnung 2 vom 13. März 2020 über Massnahmen zur Bekämpfung des Coronavirus (COVID-19-Verordnung 2) (Version vom 15. Mai 2020), S. 21.

102 Nachdem im Rahmen des Lockerungsprozesses die maximale Anzahl Personen auf 30 erhöht wurde, hat sich diese Problematik gelöst.

103 BAG (Fn. 100), 21.

104 Point de Presse Coronavirus vom 18. Mai 2020 (ab 6:25). In die Erläuterungen wurde dies jedoch nicht übernommen.
25 Neben der Organisation oder Durchführung einer verbotenen Veranstaltung ist auch die Verletzung von anderen in Art. 6 geregelten Pflichten ${ }^{105}$ tatbestandsmässig. ${ }^{106}$ Dabei kann sich nur die «verantwortliche Person» bzw. der «Organisator» strafbar machen. ${ }^{107}$ Oftmals war aber unklar, welche Handlungen strafbar waren. Im Rahmen der Lockerungen der Massnahmen wurde diese Problematik mittels präziser formulierten Tathandlungsvarianten entschärft. Es wurden aber auch neue Probleme geschaffen. So müssen die verantwortlichen Personen bzw. die Organisatoren «die Vorgaben für die Durchführung von Veranstaltungen nach Artikel 6 Absätze 35» einhalten oder umsetzen. ${ }^{108}$ Bei privaten Veranstaltungen müssen dabei «die Empfehlungen des BAG betreffend Hygiene und soziale Distanz eingehalten werden»109. Damit ergibt sich der Umfang der strafbaren Handlung erst in einer Kombination mit ausserhalb der Verordnung liegenden Empfehlungen des BAG. Mag ein solcher Verweis im Rahmen einer gesundheitspolizeilichen Massnahme zulässig sein, ${ }^{110}$ so ist eine sich darauf beziehende Strafbarkeit höchst problematisch. Dass die Ermittlung des Normgehalts bei einem Verweis auf eine Norm,

$105 \overline{\text { Art. } 6 \text { enthielt in verschiedentlich angepasster }}$ Form Anweisungen für nicht geschlossene Einrichtungen und zugelassene Veranstaltungen, bspw. die Vorschrift, ein Sicherheitskonzept vorzusehen, oder die Beschränkung der Anzahl Gäste an einem Restauranttisch (vgl. Art. 6 Abs. $3^{\text {bis }}$ [29. April 2020, AS 2020 1401]).

106 Art. $10 f$ Abs. 1 lit. a-f.

107 Seit der am 6. Juni 2020 in Kraft getretenen Fassung [AS 2020 1815] ergibt sich dies ausdrücklich aus der Bestimmung. Vorher war die Beschränkung des Täterkreises nur indirekt herzuleiten und ergab sich nicht aus dem Wortlaut der Bestimmung.

108 Art. $10 f$ Abs. 1 lit. b.

109 Art. 6 Abs. 4 lit. a.

110 Vgl. BGE 131 II 670. 
die wiederum auf Empfehlungen eines Bundesamts verweist, aufwendig und kompliziert ist, wird kaum in Abrede zu stellen sein.

Teilweise wird angenommen, dass unbestimmte Rechtsbegriffe durch eine konsistente Rechtspraxis präzisiert werden können; ${ }^{111}$ genau dies scheidet vorliegend aber aus. So ist die Notverordnung gerade darauf ausgelegt, nur für eine sehr begrenzte Zeit zu gelten. Ausserdem waren verschiedene Verhaltensweisen nur sehr kurz der Strafbarkeit unterstellt. Entsprechend ist es unmöglich, eine einheitliche Rechtspraxis zu den unbestimmten Rechtsbegriffen zu entwickeln und damit der entstehenden Rechtsunsicherheit zu begegnen. Insgesamt genügt Art. $10 f$ Abs. 1 den Anforderungen nach Art. 7 EMRK und Art. 1 StGB nicht.

\section{2. Übertretungen (Art. $10 f$ Abs. 2)}

27 Die Verordnung sieht in Art. $10 f$ Abs. 2 vier Übertretungstatbestände vor: Der Verstoss gegen das Versammlungsverbot nach Art. $7 c$ (lit. a), die Ausfuhr von Schutzausrüstung oder wichtigen medizinischen Gütern ohne Bewilligung nach Art. $4 b$ Abs. 1 (lit. b), der Verstoss gegen Einschränkungen des grenzüberschreitenden Verkehrs nach Art. 4 Abs. $4^{112}$ (lit. c) ${ }^{113}$ sowie der Verstoss gegen das Verbot des Einkaufstourismus (lit. d) ${ }^{114}$. Im Folgenden wird untersucht, ob die Übertretungstatbestände rechtmässig sind (a), welche Folgen behördliche Informationen für das Unrechtsbewusstsein haben können (b) und ob die An-

111 Vgl. Urteil des EGMR 14307/88 vom 25. Mai 1993 (Kokkinakis gegen Griechenland), Ziff. 52; BSK StGB-Popp/Berkemeier, Art. 1 N 47.

112 Aufgehoben per 15. Juni 2020 (AS 2020 2099).

113 Aufgehoben per 15. Juni 2020 (AS 2020 2099).

114 Aufgehoben per 15. Juni 2020 (AS 2020 2099). wendung des Ordnungsbussenverfahrens rechtmässig ist (c).

\section{a) Rechtmässigkeit}

28 Hinsichtlich der Verhältnismässigkeit sind die Übertretungen im Vergleich zum Vergehen weniger problematisch. ${ }^{115}$ So handelt es sich um Übertretungen, die an die im EpG vorgesehenen Taten angelehnt sind. ${ }^{116}$ Auch betreffend ihre Bestimmtheit sind die Übertretungen weniger diskussionswürdig als das Vergehen in seiner ursprünglichen Fassung. ${ }^{117}$ Einerseits sind die Anforderungen an die Bestimmtheit infolge der niedrigeren Strafdrohung weniger streng, ${ }^{118}$ anderseits erfassen die Übertretungen genauer definierte Tathandlungen. Alle vier Übertretungen verweisen auf eine Verletzung anderer Artikel der Verordnung, die klarer sind als Art. 6 und sich im üblichen Rahmen von auslegungsbedürftigen Tatbeständen halten dürften.

\section{b) Fehlendes Unrechtsbewusstsein}

29 Eine besondere Situation ergab sich dadurch, dass die Aktualisierungen und die Auslegung der Normtexte anlässlich der regelmässigen Pressekonferenzen thematisiert wurden. Dass daraus Probleme entstehen können, zeigt die Kommunikation zu Art. $10 f$ Abs. 2 lit. a. Obschon der Tatbestand vom Verbot von öffentlichen Menschenansammlungen sprach, verwies er bis zur Anpassung am 30. Mai 2020 auf eine Verletzung des gesamten Art. $7 c$, womit auch ein Verstoss gegen die 2-m-Abstandsregel der Straf-

\footnotetext{
115 S. IV.1.a.

116 Vgl. Art. 83 EpG.

117 S. IV.1.b.

118 BGE 138 IV 13 E. 4.1.
} 
barkeit unterstellt war. ${ }^{119}$ Nur wo die Abstandseinhaltung absolut unmöglich erschien, war die Abstandspflicht und damit auch die Strafbarkeit eingeschränkt. ${ }^{120}$ Entgegen Aussagen von Vertretern der Bundesbehörden ${ }^{121}$ war die Abstandsregel von dieser Ausnahme abgesehen von allen Personen einzuhalten - also auch von Familien und Personen, die im gleichen Haushalt leben.$^{122}$ Die behördliche Information dürfte dazu geführt haben, dass bei Familienmitgliedern oder im gleichen Haushalt wohnenden Personen, die sich in der Öffentlichkeit nicht an die Abstandsregeln hielten, ein Verbotsirrtum nach Art. 21 StGB bestand. Ein solcher liegt vor, wenn der Täter fälschlicherweise glaubt, sein Handeln werde von einer Verbotsnorm nicht erfasst. ${ }^{123}$ Gerade weil betroffene Personen auf Aussagen von Behörden vertrauen, dürfte der Irrtum nicht vermeidbar gewesen sein, da sich durch entsprechende Aussagen auch ein gewissenhafter Mensch hätte in die Irre führen lassen. ${ }^{124}$ Die behördliche Kommunikation

$119 \overline{\text { BAG (Fn. 101), S. } 41 \text { f. Seit der Anpassung ver- }}$ weist die Bestimmung nur noch auf eine Verletzung von Art. 7c Abs. 1, womit die Missachtung der Abstandsregeln nicht mehr bestraft werden kann, vgl. BAG (Fn. 101), S. 33.

120 BAG (Fn. 101), S. 33, nennt die Mutter mit ihrem Kleinkind oder die Frau, die ihren gehbehinderten Partner stützt.

121 Vgl. z.B. Point de Presse Coronavirus vom 18. Mai 2020 (ab 22:31) und Point de Presse Coronavirus vom 15. Mai 2020 (ab 56:22): Daniel Koch, Delegierter des BAG für COVID-19, verneinte die Geltung der Abstandsregeln für Familien ausdrücklich; vgl. auch Michael von Ledebur/Fabian Baumgartner, Händchen halten verboten? Die Polizei büsst in Zürich eine Frau, weil sie zu wenig Abstand zu ihrem Partner hielt, nzz.ch vom 15. Mai 2020.

122 So ausdrücklich BAG (Fn. 101), S. 33.

123 Sog. direkter Verbotsirrtum, s. Kurt Seelmann/Christopher Geth, Strafrecht Allgemeiner Teil, 6. Aufl., Basel 2016, Rz. 238.

124 Vgl. BGE 75 IV 150 E. 3; Seelmann/Geth (Fn. 123), Rz. 242 m.w.H. führte somit letztlich dazu, dass verschiedene strafwürdige Verhaltensweisen hätten straflos bleiben müssen.

\section{c) Anwendung des Ordnungsbussenverfahrens}

3o Für die Übertretungen nach Art. $10 f$ Abs. 2 lit. a, c und d wird das Verfahren nach Ordnungsbussengesetz ${ }^{125}$ für anwendbar erklärt, und es sind Ordnungsbussen von 100 Franken vorgesehen. ${ }^{126}$ Für die Übertretungen nach Art. 10 $f$ Abs. 2 lit. c und d wird die Eidgenössische Zollverwaltung als zuständig erklärt, Ordnungsbussen zu erheben. ${ }^{127}$

31 Das Ordnungsbussenverfahren ist ein formalisiertes, rasches und kostengünstiges Verfahren, das auf die Erledigung von leicht nachweisbaren Massendelikten zugeschnitten ist. ${ }^{128}$ Da sich das Verfahren bei der Ahndung anderer Übertretungen bewährt habe, wird es auch zur Abwicklung ausgewählter Übertretungen der COVID-19-Verordnung 2 vorgesehen. ${ }^{29}$ Das Verfahren ist dadurch gekennzeichnet, dass ausgewählte Behörden unmittelbar feststellen, dass ein Straftatbestand erfüllt ist, und dafür einen vorab bestimmten Bussbetrag verhängen.130 Dabei sind die zuständigen

$125 \overline{\text { Ordnungsbussengesetz vom 18. März } 2016 \text { (OBG; }}$ SR 314.1).

126 Art. $10 f$ Abs. 3 lit. b und Abs. 4.

127 Art. $10 f$ Abs. 5.

128 Philippe Weissenberger, Kommentar Strassenverkehrsgesetz und Ordnungsbussengesetz, Zürich 2015, Art. 1 OBG N 10; vgl. auch Urteil des Bundesgerichts 6B_722/2019 vom 23. Januar 2020 E. 1.3.1.

129 BAG, Erläuterungen zur Verordnung 2 vom 13. März 2020 über Massnahmen zur Bekämpfung des Coronavirus (COVID-19-Verordnung 2) (Version vom 2. Juni 2020), S. 41.

${ }^{130}$ Grundsätzlich ergibt sich die Höhe der Busse aus den Anhängen zur OBV (Ordnungsbussenverordnung vom 16. Januar 2019 [SR 314.11]). 
Behörden von der Anwendung der Strafzumessungsgrundsätze des Strafgesetzbuches dispensiert, ${ }^{131}$ indem dem Verschulden, dem Vorleben und den persönlichen Verhältnissen des Täters keine Bedeutung zukommt. ${ }^{132}$ Nichtsdestotrotz besteht weitgehende Einigkeit, dass es sich um eine strafrechtliche Sanktion und um ein Strafverfahren handelt, weshalb mit Ausnahme der vorbehaltenen Bestimmungen alle Grundsätze des Strafrechts und Strafprozessrechts gelten. ${ }^{133}$

Das Ordnungsbussenverfahren ist nur auf Übertretungen anwendbar, die in einem in Art. 1 Abs. 1 lit. a OBG erwähnten Gesetz oder einer sich auf eines dieser Gesetze abstützenden Verordnung aufgeführt ist. Ausserdem muss der Bundesrat die Übertretungen und den vorgesehenen Bussbetrag auflisten. ${ }^{134}$ Die entsprechenden Listen finden sich in den Anhängen zur OBV. Nachdem die Anwendung des Ordnungsbussenverfahrens mit der Änderung vom 20. März 2020 in der Verordnung festgeschrieben wurde, wurde die Liste in Anhang 2 der OBV am 27. März 2020 um die entsprechenden Tatbestände ergänzt. ${ }^{135}$ Sie werden alle in Ziff. XV aufgeführt, und die Busse be-

$131 \overline{\text { Urteil des Bundesgerichts 6B_722/2019 vom }}$ 23. Januar 2020 E. 1.3.1.

132 Art. 1 Abs. 5 OBG.

133 Urteil des Bundesgerichts 6B_722/2019 vom 23. Januar 2020 E. 1.3.1; Stefan Maeder, Sicherheit durch Gebühren?, AJP 2014, S. 679 ff., 681; Markus H. F. Mohler, Anmerkungen zur vorgeschlagenen Revision des Ordnungsbussengesetzes, Jusletter vom 10. August 2015, Rz. $11 \mathrm{ff}$. m.w.H.

134 Art. 1 Abs. 2 i.V.m. Art. 15 OBG; vgl. auch Botschaft vom 17. Dezember 2014 zum Ordnungsbussengesetz (BBl 2015 959), S. 981.

135 COVID-19-Verordnung 2 (27. März 2020 [AS 2020 1101], 1. April 2020 [AS 2020 1137] und 16. April 2020 [AS 2020 1245]). trägt CHF 100. ${ }^{136}$ Die Übertretungen der Verordnung sind damit in der Liste nach Art. 1 Abs. 2 OBG vorgesehen, stützen sich aber nicht auf ein Gesetz i.S.v. Art. 1 Abs. 1 lit. a OBG. Damit stellt sich die Frage, ob der Bundesrat die Übertretungen überhaupt dem Ordnungsbussenverfahren unterstellen durfte.

33 Im OBG wurde bewusst eine abschliessende Liste an Gesetzen aufgenommen, damit keine Unklarheiten entstehen, wann das Ordnungsbussenverfahren anwendbar ist. ${ }^{137}$ Es ist nicht ersichtlich, dass dem Bundesrat in diesem Punkt aufgrund der epidemiologischen Lage ein Regelungsspielraum zukäme. Die Anwendung des Ordnungsbussenverfahrens hat keinen Bezug zur Bekämpfung der Epidemie. ${ }^{138}$ Auch lassen sich dadurch die aufgestellten Regeln nicht effektiver durchsetzen, ${ }^{139}$ weshalb diese Regelung durch die Notverordnungskompetenz des Bundesrats nicht gedeckt ist. Schliesslich unterliegen die Übertretungen des EpG nicht dem Ordnungsbussenverfahren, 140 weswegen auch die - durch das Bundesgericht anerkannte ${ }^{141}$ - analoge Anwendung des Ordnungsbussenverfahrens ausgeschlossen ist.

\footnotetext{
136 OBV, Anhang 2, Ziff. XV, 15001-15004.

137 Botschaft OBG (Fn. 134), S. 970.

${ }_{13}^{8}$ Vgl. auch Brunner/Wilhelm/Uhlmann (Fn. 6), S. 699, die für den Ausschluss des Rechtsschutzes in Art. 11 Abs. 3 der Verordnung über die Abfederung der wirtschaftlichen Auswirkungen des Coronavirus (COVID-19) im Kultursektor vom 20. März 2020 (COVID-Verordnung Kultur; SR 442.15) zum gleichen Schluss kommen.

139 Vgl. Marcel Alexander Niggli/Stefan Maeder, Die Haftung des Motorfahrzeughalters für Ordnungsbussen, in: Probst/Werro (Hrsg.), Strassenverkehrsrechts-Tagung 2018, Bern 2018, S. 65 ff., 75 .

140 Art. 1 Abs. 1 lit. a OBG.

141 BGE 114 IV 50.
} 
34 Die Praxis zu den teilweise unklaren Übertretungen macht deutlich, dass die Anwendung des Ordnungsbussenverfahrens und die damit einhergehende Einschränkung des Rechtsschutzes ${ }^{142}$ Probleme verursacht. Das zeigt sich etwa daran, dass Verstösse gegen das Versammlungsverbot kantonal unterschiedlich geahndet wurden ${ }^{143}$ und dass wegen des Verstosses gegen die Abstandsregeln teilweise auch in einer Hausgemeinschaft lebende Personen gebüsst wurden. ${ }^{144}$ Sodann wurden von Zollbeamten in vermeintlicher Anwendung von Art. $10 f$ Abs. 2 lit. d offensichtlich rechtswidrige Bussen ausgesprochen. Gebüsst wurden auch Personen, die aus anderen Gründen als für Einkaufstourismus ins Ausland gereist waren, da sie sich «nicht im Sinne des Bundesrates» verhalten hätten. ${ }^{145}$ Solche Handlungen sind offensichtlich nicht von Art. $10 f$ Abs. 2 lit. d erfasst.

\section{Ausblick}

35 Momentan ist absehbar, dass im Rahmen von weiteren Lockerungen die Strafbestimmungen aus der COVID-19Verordnung 2 verschwinden werden. Damit darf die Thematik aber nicht vom Tisch sein. Die getroffenen Massnahmen und insbesondere der Erlass von Straf-

$142 \overline{\text { Art. } 11 \text { OBG: Bezahlte Bussen erwachsen direkt in }}$ Rechtskraft.

143 Vgl. Iole Fargnoli/Maria Lapadula, Nicht mehr als fünf, Jusletter vom 25. Mai 2020, Rz. $8 \mathrm{ff}$.

144 Von Ledebur/Baumgartner (Fn. 121). Unverständlich und das Problem verstärkend war, dass der Bundesrat als Reaktion auf diese Vorkommnisse nicht die Verordnung präzisierte, sondern die Polizei anwies, bei Personen, die im gleichen Haushalt wohnen, Augenmass walten zu lassen und vom strafprozessualen Opportunitätsprinzip nach Art. 8 StPO Gebrauch zu machen; vgl. BAG (Fn. 101), S. 33.

145 srf.ch vom 8. Mai 2020 (Überschreiten Grenzwächter ihre Grenzen?). vorschriften müssen im Nachgang genau analysiert werden, damit Lehren für ähnliche Ereignisse in der Zukunft gezogen werden können.

36 Der Bundesrat hat sich teilweise fast nonchalant über gesetzliche Erfordernisse und Voraussetzungen hinweggesetzt. Er hat unverhältnismässige Strafen vorgesehen für unbestimmte Verhaltensweisen, die teilweise in einem Verfahren abgehandelt wurden, das eine erhebliche Einschränkung des Rechtsschutzes bedeutet und sich auf keine rechtliche Grundlage stützen kann. Es ist deshalb kritisch zu reflektieren, unter welchen Voraussetzungen der Bundesrat in einer ausserordentlichen Lage legitimiert ist, Strafvorschriften zu erlassen, und ob er darin höhere Strafdrohungen vorsehen kann als das Gesetz. Auch muss überlegt werden, wie Strafvorschriften in einem sich rasch ändernden Umfeld auszugestalten sind, sodass sie dennoch den Anforderungen an die Bestimmtheit genügen. Weiter hat sich gezeigt, wie wichtig während einer Krise eindeutige Kommunikation der Behörden ist.

37 Gleichzeitig muss der Gesetzgeber das Geschehene und insbesondere die Massnahmen des EpG genauer anschauen. Bedarf es im Gesetz einer Einschränkung, wozu der Bundesrat im Falle einer ausserordentlichen Lage legitimiert ist? Gerade im Hinblick auf das Legalitätsprinzip wäre es wünschenswert, dass der Gesetzgeber die Strafvorschriften des EpG erweitert, damit sie auch auf Massnahmen anwendbar sind, die der Bundesrat in einer ausserordentlichen Lage erlässt. Vor dem Hintergrund der Corona-Krise ist auch die Angemessen- 
heit der bestehenden Strafnormen und Strafdrohungen zu prüfen. ${ }^{146}$

38 Es ist zentral, dass der bundesrätliche Umgang mit Strafvorschriften in dieser Pandemie genau aufgearbeitet wird, um für zukünftige Krisensituationen gerüstet zu sein. Nur so kann erreicht werden, dass das Strafrecht in der Krise nicht das Strafrecht in die Krise stürzt.

146 Bereits vor der Covid-19-Pandemie für schärfere Sanktionen plädierend Nathalie Dongois, Quelle répression pénale en cas de transmission du VIH par voie sexuelle?, ZStrR 2015, S. 42 ff., 6o, die als Beispiel die Verursachung eines EbolaAusbruchs nennt. 